\title{
Fatigue syndrome in sarcoidosis
}

The authors declare no financial disclosure

\begin{abstract}
Sarcoidosis is an inflammatory disease of unknown etiology. Most commonly it results in the formation of non-caseating granulomas in intrathoracic lymph nodes and lung parenchyma, but the clinical course and picture may be complicated by extrapulmonary involvement and many non-respiratory signs and symptoms which are directly related to the disease. In addition, sarcoidosis patients may suffer from a plethora of symptoms of uncertain or unknown origin. Fatigue is one of these symptoms, and according to some authors it is reported by the majority of patients with active sarcoidosis, but also by a smaller proportion of patients with inactive sarcoidosis, or even with complete clinical and radiological remission. Therefore the term fatigue syndrome is frequently used to name this clinical problem. The definition of fatigue syndrome in sarcoidosis is imprecise and the syndrome is usually recognized by use of validated questionnaires. In this review the uptodate knowledge in this field was presented and different challenges connected with this syndrome were described.
\end{abstract}

Key words: sarcoidosis, fatigue, diagnosis, treatment

Pneumonol Alergol Pol 2016; 84: 244-250

\section{Introduction}

Sarcoidosis is a multisystemic disease of unknown etiology, characterized by formation of non-caseating granulomas in different organs. Most frequently the disease presents with enlarged intrathoracic lymph nodes and/or lung parenchymal infiltrations. It has been estimated to be the second frequent chronic respiratory disease among young adults ( $<40$ year of life), after asthma. Prevalence may be different in different ethnic groups, and is estimated to $5-40 / 100,000$. In some European countries (ie Denmark) its incidence is close to $5 / 100,000 /$ year [1-4].

Extrathoracic manifestations can result either from direct organ involvement (most frequently skin, peripheral lymph nodes, eyes, liver, spleen, but all organs may be involved). Many general or non-respiratory symptoms may be unrelated to specific organ involvement. For instance, patients with altered vitamin D metabolism and hypercalciuria may suffer from nephrolithiasis or symptoms related to high serum calcium concentration. The plethora of non-specific general or non-respiratory symptoms unrelated to specific organ involvement include fever, weight loss, myalgia, arthralgia, sweating, general malaise, depression, and fatigue. The origin and etiology of these symptoms is largely unknown.

Chronic fatigue is one of the symptoms most frequently reported by sarcoidosis patients. Interestingly, it may appear both in the course of active sarcoidosis as well as in those with disease remission. Its frequency is estimated at $50-70 \%$ of all sarcoidosis patients [2, 5, 6]. It is associated with decreased quality of life and quite often with depression [5-7]. In 1994 American Centers for Disease Control and Prevention (CDC) defined chronic fatigue syndrome (CFS) as a "debilitating and complex disorder characterized by intense fatigue that is not improved by bed rest and that may be worsened by physical activity or mental exertion. People with CFS often function at a substantially lower level of

Address for correspondence: Wojciech J. Piotrowski, Department of Pneumonology and Allergy, Medical Univeristy of Lodz, e-mail: wojciech.piotrowski@umed.lodz.pl DOI: 10.5603/PiAP.2016.0030

Received: 15.05 .2016

Copyright (C) 2016 PTChP

ISSN 0867-7077 
activity than they were capable of before they became ill" [8].

The document further precise, that the diagnosis requires three criteria:

1) The individual has had severe chronic fatigue for 6 or more consecutive months that is not due to ongoing exertion or other medical conditions associated with fatigue (these other conditions need to be ruled out);

2) The fatigue significantly interferes with daily activities and work;

3) The individual concurrently has 4 or more of the following 8 symptoms:

- post-exertion malaise lasting more than 24 hours,

- unrefreshing sleep,

- significant impairment of short-term memory or concentration,

- muscle pain,

- pain in the joints without swelling or redness,

- headaches of a new type, pattern, or severity,

- tender lymph nodes in the neck or armpit,

- a sore throat that is frequent or recurring.

These symptoms should have persisted or recurred during 6 or more consecutive months of an illness and they cannot have first appeared before the fatigue [cited from 8].

These criteria describe fatigue syndrome, regardless the reason. The syndrome has been described in a variety of different clinical contexts, for instance in patients infected with various viruses (Ebstein-Bar, human immunodeficiency virus, hepatitis $\mathrm{C}$ ), neoplastic disease, rheumatoid arthritis and systemic sclerosis, multiple sclerosis, fibromyalgia, obstructive sleep apnea and many others $[8,9]$.

\section{Types of fatigue in sarcoidosis patients}

Sharma [10] depicted four types of fatigue in patients with sarcoidosis:

1) Early-morning fatigue - when the patient is not able to arise or arises with the feeling of inadequate sleep;

2) Intermittent fatigue - when the patient wakes up in good condition and feeling of adequate sleep rest, but after few hours of daytime activity become tired and exhausted. After an hour or more is able to restart his/her activity;

3) Afternoon fatigue - the patient wakes up refreshed and full of energy, but in the early afternoon feels exhausted, and this feeling continues until next morning;
4) Post-sarcoidosis chronic fatigue syndrome which occurs in patients who are evidently free of disease, with clinical and radiological signs of remission.

De Kleijn et al. [11] examined 434 patients with chronic sarcoidosis, and divided the whole cohort into three groups:

1) Mild fatigue - patients with no fatigue or with only mild symptoms;

2) Intermittent fatigue - described in a similar way as by Sharma [10];

3) All-day fatigue - when the feeling of fatigue is constant and lasts the whole day.

Because the authors have not found patients with remission of sarcoidosis in their study group (only active sarcoidosis patients were included), they could not confirm the presence of post-sarcoidosis fatigue syndrome. They also have not found any patients matching to afternoon fatigue type described by Sharma [10]. In our practice the post-sarcoidosis fatigue syndrome happens quite frequently. It is a real challenge for researchers and practitioners, as the definition and criteria are even more vague, than in case of fatigue associated with any other active chronic disease. Sharma estimated that it occurs in about $5 \%$ of sarcoidosis patients [10].

\section{Different measures to estimate fatigue}

In face of a variety of different types of fatigue and a long list of chronic diseases and states, that can be accompanied by fatigue, many different questionnaires have been used for fatigue assessment [9]. Questionnaires have the advantage over the simple yes-or-no response to the question asked to the patient about the feeling of fatigue, and allow objective assessment of fatigue severity. In fact, other objective tests are unknown, therefore the diagnosis of fatigue syndrome needs to be based on questionnaires, with an estimated threshold cut-off value which is believed to differentiate those suffering from clinically significant fatigue form fatigue-free individuals. Many of them have been tried also in sarcoidosis. The only validated method of its evaluation in this special group of patients is Fatigue Assessment Scale (FAS) [12]. This scale consists of 10 questions specific for sarcoidosis-related fatigue, five of them describing physical and five - mental fatigue (Table 1). A value of 22 and above points has been set up to discriminate patients with from those without significant fatigue [12]. It has been shown in various clinical contexts, that FAS is an unidimentional instrument (describes fatigue quantitatively and 


\section{Table 1. Questions composing the Fatigue Assessment Scale (FAS). Each question is evaluated in a 5-point scale: never - 1; sometimes - 2; regularly -3 ; often -4 ; always -5}

1. I am bothered by fatigue

2. I get tired very quickly

3. I don't do much during the day

4. I have enough energy for everyday life

5. Physically, I feel exhausted

6. I have problems to start things

7. I have problems to start things

8. I feel no desire to do anything

9. Mentally, I feel exhausted

10. When am doing something, I can concentrate quite well

independently of other confounders, such as depression) [13, 14]. This feature was also confirmed in a population of sarcoidosis patients [12]. The change of 4 points in a certain time is considered a minimal clinically important difference [15]. In fact, FAS is the most important method to diagnose fatigue and to estimate its severity.

Other scales used for the multidimentional assessment of sarcoidosis-related fatigue include: WHOQOL-100 - World Health Organization Quality of Life Assessment Instrument-100 [12], MFI - Multidimentional Fatigue Inventory [16], FACIT-F - Functional Assessment of the Chronic Illness Therapy-Fatigue [17], SHQ - Sarcoidosis Health Questionnaire, SF-36 [18], CRDQ — Chronic Respiratory Disease Questionnaire [12]. BDI Beck Depression Inventory score [12] may be used for the assessment of accompanying depression, and may be helpful in differentiating fatigue from depression-related symptoms. Borg Scale and MRC - Medical Research Council scale may be used for the assessment of dyspnea and exercise intolerance, which frequently accompany fatigue [19].

\section{Possible causes of fatigue in sarcoidosis}

Factors responsible for fatigue in patients with sarcoidosis has not been defined. It is presumed, that the etiology is multifactorial and related not only to chronic systemic inflammation but also to other factors, for instance to comorbidities, treatment and psychological factors.

\section{Inflammation}

In early active phase of sarcoidosis the CD4 lymphocytes predominance is observed, and lym- phocytes differentiate to Th1 immunophenotype, which is characterized by production of specific cytokines, such as interleukin (IL)-2 and interferon (INF) $-\gamma$. Activated macrophages produce - in response to these cytokines - large amounts of tumor necrosis factor (TNF)- $\alpha$. During recovery the balance between Th1 and Th2 immunophenotypes reverses. Interestingly, in patients with post-sarcoidosis fatigue syndrome, Korenromp et al. [20] observed decreased production of Th2-related cytokines (IL-4, IL-5, IL-10) and decreased concentration of monocyte chemotactic protein (MCP)-1, also referred to as chemokine (C-C motif) ligand 2 (CCL2), which is responsible for activation of Th2 lymphocytes. Increased Th2 response in sarcoidosis remission is supposed to "calm-down" the inflammatory reaction. Therefore, inadequate Th1 to Th2 re-shifting may contribute to fatigue in these patients. The same authors in a separate study found lower ACTH and cortisol serum concentrations in patients with post-sarcoidosis fatigue than patients in remission who do not report fatigue [21]. They also reported on the specific personality profile with profound neurotic characteristics in combination with high levels of psychological distress [21]. The authors propose, that these findings should be taken into account when different treatment options are considered. Baydur et al. [22] studied relations between fatigue and baseline (resting) and post-exercise serum levels of TNF- $\alpha$ and IL- $1 \beta$ in sarcoidosis patients comparing to healthy controls. They found that patients exhibited greater fatigue, reduced cardiopulmonary function, higher MRC dyspnea scores and higher TNF- $\alpha$ plasma levels at all time-points. In treated patients only, pre-exercise plasma IL- $1 \beta$ correlated with physical fatigue, reduced motivation and total fatigue, and TNF- $\alpha$ levels correlated with general fatigue score. The authors do not answer, whether these findings were related to the treatment or to subclinical inflammation related to the mere disease [22]. Inflammation is however, an unlikely reason of fatigue in patients with sarcoidosis remission.

\section{Muscle weakness}

Muscle weakness is a frequent complaint in patients suffering from sarcoidosis [23]. Study performed among sarcoidosis patients showed, that quadriceps muscle strength and handgrip force were not decreased, but most of these patients did not complain on fatigue [24]. In many studies on patients with active sarcoidosis however, fatigue was associated with reduced muscle strength and exercise intolerance [25, 26]. There 
can be few possible causes of muscle weakness in patients suffering from sarcoidosis, starting from formation of granulomas in skeletal muscles, through steroid myopathy, and increased levels of inflammatory cytokines, to decreased physical condition related to chronicity of the disease. It is of note, that muscle sarcoidosis (granulomas found at muscle biopsy) is a rarity. Regardless the reason, the link between muscle weakness and fatigue in sarcoidosis patients still remains undefined.

\section{Lung function impairment}

Fatigue itself may negatively impact exercise tolerance. On the other hand, the influence of functional impairment on the occurrence and severity of fatigue have been assessed in few trials. Zieleźnik et al. [19] examined 76 patients with inactive sarcoidosis, and fatigue was reported by $50 \%$ of them, with almost $7 \%$ reporting severe fatigue. The authors found weak correlation between fatigue and female gender and older age, but did not find any correlation between fatigue and spirometry parameters and the distance in 6-minutes walking test.

\section{Psychological aspects}

Sarcoidosis, as a chronic disease of uncertain long term prognosis may influence the psychological aspects of patient's life, leading to depression and decreased quality of life. Chang et al. [27] reported depression in $60 \%$ of sarcoidosis patients, and showed correlations with female gender, economical status, access to health service and disease severity. Goracci et al. [28] confirmed strong relationship between sarcoidosis and depression, and its negative impact on quality of life. De Klejn et al. [29] found, that fatigue is related to depression in $34-36 \%$ and to anxiety in $43-46 \%$ of patients. Similarly, Holas et al. reported on depression in $29 \%$ and anxiety in $31 \%$ of patients with active sarcoidosis [30]. Patients presenting all-day fatigue reported more symptoms linked to depression. The study also showed, that fatigue is frequently reported by patients who do not have depression at all. In few studies it was also shown, that depression negatively impacts fatigue [31, 32]. It seems probable, that depression and fatigue are strictly related to each other. Depression may increase the feeling of fatigue, general reluctance to effort, aversion to treatment and regular medical check-ups, and may worsen the cooperation between the patient and his doctor. All these may lead to inadequate control of the disease. Sarcoidosis itself, as a chronic disease frequently severely complicated and with unpredictable prognosis may worsen depressive symptoms.

\section{The influence of treatment, comorbidities and extrapulmonary locations}

Fleisher et al. in the German study [33] of over 1000 patients with sarcoidosis proved the influence of several different factors on the severity of fatigue. They reported, that patients with muscle, bone and neural involvement presented more severe fatigue than patients with pulmonary sarcoidosis. In general, patients with extrapulmonary sarcoidosis have more severe fatigue than patients with isolated pulmonary sarcoidosis. In addition, the authors showed, that treatment with systemic steroids and methotrexate is correlated with more severe fatigue. There was a strong correlation between fatigue and comorbidities, such as systemic hypertension, thyroid diseases, obesity, restless legs syndrome, and diabetes. But the most strongly associated comorbidities with fatigue were pulmonary hypertension and sleep apnoe syndrome. Sleep apnoe syndrome is several times more frequent in patients with sarcoidosis than in healthy population, and may lead to fatigue, anxiety and depression [34, 35].

Many patients with sarcoidosis suffer from small fiber neuropathy (SFN), which affects small peripheral nerve fibers and manifests as severe hyperaesthesia, pain, numbness of distal parts of extremities and is associated with autonomic dysfunction. TNF- $\alpha$ may be a crucial cytokine in the pathogenesis of SFN [36]. Dysautonomias are frequently associated with chronic fatigue [37]. Therefore it may be presumed, that treatment of sarcoidosis patients suffering from chronic fatigue with anti-TNF- $\alpha$ agents [38] or intravenous immunoglobulin [39] may be effective not only to alleviate symptoms of neuropathy, but also to cure fatigue.

\section{Attempts of therapy}

There is no single therapy for chronic fatigue syndrome in the course of sarcoidosis verified and evaluated in randomized trials. Although some authors, based on their personal experience and confidence suggested to treat these patients with low-dose systemic steroids and/or hydroxychlorochine [10], there are no evidence-based data to prove the efficiency of this regimen. From other sources we can learn, that treatment with steroids [12] and immunosuppressive drugs [40] may be a causative factor of fatigue. Therefore, it seems 
reasonable to advise to follow the general rules which define indications to systemic therapy in sarcoidosis [1]. According to these rules, fatigue is not an indication for systemic steroids and immunosuppressive drugs.

Due to multifactorial etiology and complex pathogenesis no single method of treatment exists. The majority of trials performed in the indication of fatigue in a plethora of different clinical backgrounds concerned neurostimulants and anti-TNF therapy [41-44].

Metylfenidate represents a group of drugs called neurostimulants, and is used in the treatment of attention deficit, hyperactivity disorder (ADHD), and narcolepsy. It has been also used as an adjunctive therapy of depression $[45,46]$. Another neurostimulant, modafinil is a dopamine re-uptake inhibitor used in the treatment of narcolepsy. Modafinil or its R isomere, armodafinil has been used to treat excessive sleepness in the course of sleep apnea syndrome [47, 48], and narcolepsy [49]. The drug has been also used to treat fatigue associated with depression [50] and multiple sclerosis [51, 52].

There are only few completed trials in sarcoidosis-related chronic fatigue syndrome. Wagner et al. [53], in an open-label study treated five patients with sarcoidosis who presented chronic fatigue with methylphenidate (MPH), and the treatment was effective in four of them. In another study dexmethylphenidate (d-MPH) was used to treat fatigue in a double-blind randomized, placebo-controlled, cross-over trial. The study included 10 patients with severe sarcoidosis, all patients were receiving systemic therapy. The drug was well tolerated and substantial improvement of fatigue was noticed in all treated patients [54]. It is not clear what is the exact mechanism of this positive drug effect. It cannot be excluded, that it is connected with its antidepressive activity.

Armodafinil was used in one doble-blind, placebo-controlled, cross-over study [55]. The authors recruited 15 patients with sarcoidosis-related fatigue, in whom sleep apnea was excluded. The treatment significantly reduced fatigue scores assessed by FAS and FACIT-F questionnaires, even in patients who did not have excessive daytime somnolence.

All these studies were performed in small study groups, which is an obvious limitation. However, the results are promising and should encourage researchers to design a big multinational trial in order to confirm the effectiveness of neurostimulants in the treatment of sarcoidosis-related fatigue.
TNF- $\alpha$ is a key cytokine in the pathogenesis of sarcoid inflammation, and anti-TNF drugs are accepted in the treatment of refractory and difficult-to-treat sarcoidosis [56, 57]. Chronic persisted inflammation may play a role in the pathogenesis of fatigue in sarcoidosis [58]. Data on the influence of anti-TNF therapy on sarcoidosis-related fatigue are scarce. Elfferich et al. [59] observed prospectively 343 patients with sarcoidosis, some of them with indications for systemic therapy (steroids, methotrexate, anti-TNF). They registered the cognitive impairment and fatigue at baseline and after 6 months follow-up, and they found, that only patients treated with anti-TNF drugs showed substantial improvement of cognition and fatigue.

There is no doubt, that the treatment of fatigue related to sarcoidosis should be multidisciplinary. The proper therapy adjusted to clinical presentation, disease phenotype and severity should be introduced in selected patients in order to alleviate symptoms and prevent disease progression. Treatment of comorbidities, psychotherapy, physical exercises and rehabilitation seems to be equally important.

\section{Summary}

Chronic fatigue is a very frequent symptom reported by sarcoidosis patients. It may affect up to $70 \%$ of patients. Due to its severity and diurnal profile several types of fatigue have been described, starting from mild intermittent to constant fatigue. The most intriguing is the occur-

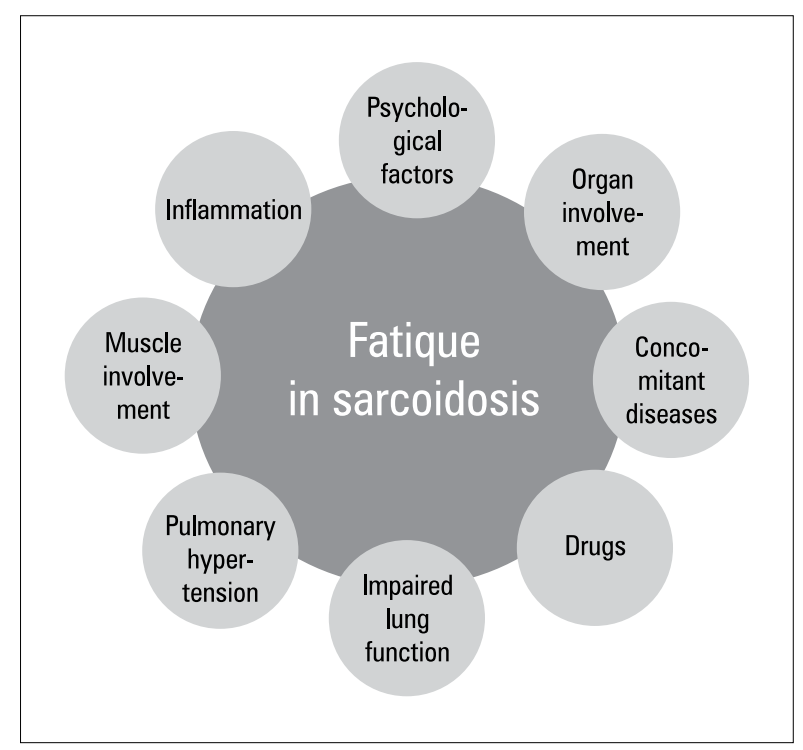

Figure 1. Possible factors involved in the pathogenesis of fatigue in sarcoidosis 
rence of fatigue in some patients with sarcoidosis remission. Chronic fatigue negatively impacts patient's quality of life, increases depression and anxiety. The etiology remains unknown, but it has been postulated to occur more frequently in those with sustained systemic inflammation, decreased muscle strength, extrapulmonary presentations, treated with steroids and immunosuppressive drugs, with comorbidities and coexistent depression (Fig. 1). Effective treatment is unknown, however neurostimulants and anti-TNF agents have been shown effective in small preliminary trials. Psychotherapy and rehabilitation may be also important, however they have not been studied in this clinical context. Further trials of improved quality, involving higher numbers of patients and designed specifically for sarcoidosis-related fatigue should be planned.

\section{Conflict of interest}

\section{The authors declare no conflict of interest.}

\section{References:}

1. Hunninghake GW, Costabel U, Ando M et al. ATS/ERS/WASOG statement on sarcoidosis: American Thoracic Society/ European Respiratory Society/World Association of Sarcoidosis and other Granulomatous Disorders. Am J Respir Crit Care Med 1999; 160: 736-755.

2. Iannuzzi MC, Rybicki BA, Teirstein AS. Sarcoidosis. N Engl J Med 2007; 357: 2153-2165.

3. Wirnsberger RM, De Vries J, Wouters EFM, Drent M. Clinical presentation of sarcoidosis in The Netherlands An epidemiological study. Neth J Med 1998; 53: 53-60.

4. European Lung White Book 2015. http://www.erswhitebook. org/chapters/interstitial-lung-diseases/; 28.01.2016.

5. Korenromp I, Van Den Bosch J, Grutters J, Vogels O, Heijnen C. Characterization of chronic fatigue in patients with sarcoidosis in clinical remission. Chest 2011; 140: 441-447.

6. Michielsen HJ, Drent M, Peros-Golubicic T, De Vries J. Fatigue is associated with quality of life in sarcoidosis patients. Chest 2006; 130: 989-94.

7. Chang B, Steimel J, Moller DR et al. Depression in sarcoidosis. Am J Respir Crit Care Med 2001; 163: 329-34.

8. Centers for Disease Control and Prevention. http://www.cdc. gov/cfs/case-definition/; 28.01.2016.

9. Whitehead L. The measurement of fatigue in chronic illness: A systematic review of unidimentional and multidimentional fatigue measures. J Pain Symptom Manage 2009; 37: 107-128.

10. Sharma OP. Fatigue and sarcoidosis. Eur Respir J 1999; 13: 713-714.

11. de Kleijn WP, Drent M, Vermunt JK, Shigemitsu H, De Vries J. Types of fatigue in sarcoidosis patients. J Psychosom Res 2011; 71: 416-422.

12. De Vries J, Michielsen H, Van Heck GL, Drent M. Measuring fatigue in sarcoidosis: the Fatigue Assessment Scale (FAS). Br J Health Psychol 2004; 9: 279-291.

13. Michielsen HJ, De Vries J, Van Heck GL. Psychometric qualities of a brief self-rated fatigue measure: The Fatigue Assessment Scale (FAS). J Psychosom Res 2003; 54: 345-352.

14. Michielsen HJ, De Vries J, Van Heck GL, Van De Vijver EJR, Sijtsma K. Examination of the dimentionality of fatigue: The construction of the Fatigue Assessment Scale (FAS). Eur J Psychol Assess 2004; 20: 39-48.

15. De Kleijn WPE, De Vries J, Wijnen PAHM, Drent M. Minimal (clinically) important differences for the Fatigue Assessment Scale in sarcoidosis. Respir Med 2011; 105: 1388-1395.
16. Fleischer M, Hinz A, Brähler E, Wirtz H, Bosse-Henck A. Factors associated with fatigue in sarcoidosis. Respir Care 2014; 59: 1086-1094.

17. Lower EE, Harman S, Baughman RP. Double-blind, randomized trial of dexmethylphenidate hydrochloride for the treatment of sarcoidosis-associated fatigue. Chest 2008; 133: 1189-1195. doi: 10.1378/chest.07-2952.

18. De Boer S, Wilsher ML. Validation of the Sarcoidosis Health Questionnaire in a non-US population. Respirology 2012; 17: 519-524. doi: 10.1111/j.1440-1843.2012.02134.x.

19. Zieleźnik K, Jastrzębski D, Ziora D. Fatigue in patients with inactive sarcoidosis does not correlate with lung ventilation ability or walking distance. Pilot Study. Pneumonol Alergol Pol 2015; 83: 15-22. doi: 10.5603/PiAP.2015.0002.

20. Korenromp I, Grutters J, van den Bosch J, Zanen P, Kavelaars A, Heijnen C. Reduced Th2 cytokine production by sarcoidosis patients in clinical remission with chronic fatigue. Brain Behav Immun 2011; 25: 1498-1502.

21. Korenromp IH, Grutters JC, van den Bosch JM, Heijnen CJ. Post-inflammatory fatigue in sarcoidosis: Personality profiles, psychological symptoms and stress hormones. J Psychosom Res Research 2012; 72: 97-102.

22. Baydur A, Alavy B, Nawathe A, Liu S, Louie S, Sharma O. Fatigue and plasma cytokine concentrations at rest and during exercise in patients with sarcoidosis. Clin Respir J 2011; 5: 156-164.

23. Miller A, Brown LK, Sloane MF et al. Cardiorespiratory responses to incremental exercise in sarcoidosis patients with normal spirometry. Chest 1995; 107: 323-329.

24. Wirnsberger M, Drent M, Hekelaar N et al. Relationship between muscle function and quality of life in sarcoidosis. Eur Respir J 1997; 10: 1450-1455.

25. Spruit M, Thomeer M, Decramer M et al. Skeletal muscle weakness in patients with sarcoidosis and its relationship with exercise intolerance and reduced health status. Thorax 2005; 60: 32-38.

26. Marcellis R, Lenssen A, Kleynen S, De Vries J, Drent M. Exercise capacity, muscle strength and fatigue in sarcoidosis. Eur Respir J 2011; 38: 628-634.

27. Chang B, Steimel J, Moller DR et al. Depression in sarcoidosis. Am J Respir Crit Care Med 2001; 163: 329-334.

28. Goracci A, Fagiolini A, Rottoli P et al. Quality of life, anxiety and depression in Sarcoidosis. Gen Hosp Psychiatry 2008; 30: 441-445.

29. de Kleijn W, Drent M, De Vries J, Nature of fatigue moderates depressive symptoms and anxiety in sarcoidosis, Brit J Health Psych 2013; 18: 439-452.

30. Holas P, Krejtz I, Urbankowski T, Skowyra A, Ludwiniak A Domagala-Kulawik J. Anxiety, its relation to symptoms severity and anxiety sensitivity in sarcoidosis. Sarcoidosis Vasc Diffuse Lung Dis 2013; 30: 282-288.

31. de Kleijn W, De Vries J, Lower E, Elfferich M, Baughman R, Drent M. Fatigue in sarcoidosis: a systematic review. Curr Opin Pulm Med 2009; 15: 499-506.

32. Fukuda K, Straus SE, Hickie I, Sharpe MC, Dobbins JG, Komaroff A. The chronic fatigue syndrome: a comprehensive approach to its definition and study. Ann Intern Med 1994; 121: 953-959.

33. Fleischer M, Hinz A, Brähler E, Wirtz H, Bosse-Henck A. Factors Associated With Fatigue in Sarcoidosis. Respir Care 2014; 59: 1086-1094.

34. Bosse-Henck A, Wirtz H, Hinz A. Subjective sleep quality in sarcoidosis. Sleep Medicine 2015; 16: 570-576.

35. Turner G, Lower E, Corser B, Gunther K, Baughman R. Sleep apnea in sarcoidosis. Sarcoidosis Vasc Diffuse Lung Dis 1997; 14: 61-64.

36. Heij L, Dahan A, Hoitsma E. Sarcoidosis and pain caused by small-fiber neuropathy. Pain Res Treat 2012; 2012: 256024. doi: $10.1155 / 2012 / 256024$

37. Goldstein DS, Robertson D, Esler M et al. Dysautonomias: clinical disorders of the autonomic nervous system. Ann Intern Med 2002; 137: 753-763.

38. Hoitsma E, Faber CG, van Santen-Hoeufft M, De Vries J, Reulen JP, Drent M. Improvement of small fiber neuropathy in a sarcoidosis patient after treatment with infliximab Sarcoidosis Vasc Diffuse Lung Dis 2006; 23: 73-77. 
39. Parambil JG, Tavee JO, Zhou L, Pearson KS, Culver DA. Efficacy of intravenous immunoglobulin for small fiber neuropathy associated with sarcoidosis. Resp Med 2011; 105: 101-105.

40. Love RR, Leventhal H, Easterling DV, Nerenz DR. Side effects and emotional distress during cancer chemotherapy. Cancer 1989; 63: 604-612.

41. Brown JN, Howard CA, Kemp DW. Modafinil for the treatment of multiple sclerosis-related fatigue. Ann Pharmacother 2010; 44: 1098-1103.

42. Minton O, Richardson A, Sharpe M, Hotopf M, Stone P. Drug therapy for the management of cancer-related fatigue. Cochrane Database Syst Rev 2010; 7: CD006704. doi: 10.1002/14651858. CD006704.pub3.

43. Wallace AE, Kofoed LL, West AN. Double-blind, placebocontrolled trial of methylphenidate in older, depressed, medically ill patients. Am J Psychiatry 1995; 152: 929-931.

44. Homsi J, Nelson KA, Sarhill N et al. A phase II study of methylphenidate for depression in advanced cancer. Am J Hosp Palliat Care 2001; 18:403-407.

45. Lavretsky H, Park S, Siddarth P, Kumar A, Reynolds III C. Methylphenidate enhanced antidepressant response to citalopram in the elderly: a double-blind, placebo-controlled pilot trial. Am J Geriatr Psychiatry 2006; 14: 181-185.

46. Patkar AA, Masand PS, Pae CU et al. A randomized, double-blind, placebo-controlled trial of augmentation with an extended release formulation of methylphenidate in outpatients with treatment-resistant depression. J Clin Psychopharmacol 2006; 26: 653-656.

47. Roth T, Rippon GA, Arora S. Armodafinil improves wakefulness and long-term episodic memory in nCPAP-adherent patients with excessive sleepiness associated with obstructive sleep apnea. Sleep Breath 2008; 12: 53-62.

48. Hirshkowitz M, Black J, Wesnes K, Niebler G, Arora S, Roth T. Adjunct armodafinil improves wakefulness and memory in obstructive sleep apnea/hypopnea syndrome. Resp Med 2007; 101: 616-627.

49. Harsh JR, Hayduk R, Rosenberg R et al. The efficacy and safety of armodafinil as treatment for adults with excessive sleep- iness associated with narcolepsy. Curr Med Res Opin 2006; 22: 761-774.

50. DeBattista C, Doghramji K, Menza MA, Rosenthal MH, Fieve RR. Adjunct modafinil for the short-term treatment of fatigue and sleepiness in patients with major depressive disorder: a preliminary double-blind, placebo-controlled study. J Clin Psychiatry 2003; 64: 1057-1064.

51. Zifko UA, Rupp M, Schwarz S, Zipko HT, Maida EM. Modafinil in treatment of fatigue in multiple sclerosis. Results of an open-label study. J Neurol 2002; 249: 983-987.

52. Rammohan KW, Rosenberg JH, Lynn DJ, Blumenfeld AM, Pollak CP, Nagaraja HN. Efficacy and safety of modafinil (Provigil) for thetreatment of fatigue in multiple sclerosis: a two centre phase 2 study. J Neurol Neurosurg Psychiatry 2002; 72: 179- 183.

53. Wagner MT, Marion SD, Judson MA. The effects of fatigue and treatment with methylphenidate on sustained attention in sarcoidosis. Sarcoidosis Vasc Diffuse Lung Dis 2005; 22: 235.

54. Lower EE, Harman S, Baughman RP. Double-blind, randomized trial of dexmethylphenidate hydrochloride for the treatment of sarcoidosis-associated fatigue. Chest. 2008; 133: 1189-1195. doi: 10.1378/chest.07-2952.

55. Lower EE, Malhotra A, Surdulescu V, Baughman RP. Armodafinil for sarcoidosis-associated fatigue: a double-blind, placebo-controlled, crossover trial. J Pain Symptom Manage 2013; 45: 159-169. doi: 10.1016/j.jpainsymman.2012.02.016.

56. Baughman RP, Drent M, Kavuru M et al. Infliximab therapy in patients with chronic sarcoidosis and pulmonary involvement. Am J Respir Crit Care Med 2006; 174: 795-802.

57. Baughman RP, Lower EE, Drent M. Inhibitors of tumor necrosis factor (TNF) in sarcoidosis: who, what, and how to use them. Sarcoidosis Vasc Diffuse Lung Dis 2008; 25: 76-89.

58. Drent M, Wirnsberger R, Wouters E, Schols A, Van Dieijen-Visser M, De Vries J. Association of fatigue with an acute phase response in sarcoidosis. Eur Respir J 1999; 13: 718-722.

59. Elfferich M, Nelemans PJ, Ponds RW, De Vries J, Wijnen P, Drent M. Everyday cognitive failure in sarcoidosis: the prevalence and the effect of anti-TNF-a treatment. Respiration 2010; 80: 212-219. 